Civilizar: Ciencias Sociales y Humanas 18 (35): 163-176, julio-diciembre de 2018 DOI: http://dx.doi.org/10.22518/usergioa/jour/ccsh/2018.2/a12

\title{
Hallazgos y discursos en torno a la maternidad adolescente ${ }^{1}$
}

\author{
Findings and discourses about teenage motherhood
}

Recibido: 2 de enero de 2017 - Revisado: 10 de octubre de 2017 - Aceptado: 6 de abril de 2018.

\section{Dra. Bélgica Romero-de-Loera ${ }^{2}$}

\section{Resumen}

Este artículo busca hacer visibles los estudios actuales más representativos sobre "maternidad temprana", con la finalidad de situar a las madres jóvenes en los debates socioculturales de la actualidad, identificando lo que se conoce sobre ellas, desde dónde se les ha estudiado y cuáles son las preguntas que surgen a partir de cada uno de los estudios revisados; presentando un antecedente de investigación en el contexto urbano mexicano. Los resultados de la revisión han sido categorizados temáticamente, se incluye un resumen de los cuerpos teóricos y metodológicos de las investigaciones revisadas y se concluye que el estudio de la maternidad adolescente como problema social, parte de la visión adultocéntrica de la maternidad y de la juventud.

\section{Palabras clave}

Maternidad, juventud, investigaciones sociales, salud pública.

\begin{abstract}
The objectives of this article are, on the one hand, to make visible the most representative current studies on "early motherhood", in order to situate young mothers in the sociocultural debates of today, identifying what is known about them, from where they have been seen and which questions are emerging from each of the studies reviewed. The results of the review of research articles have been thematically categorized, also included is a summary of the theoretical and methodological mainstream of the revised researchers. It is concluded that the study of adolescent motherhood as a social problem focuses on an adult-centric vision of motherhood and youth.
\end{abstract}

\section{Keywords}

Motherhood, youth, social research, public health.

\footnotetext{
${ }^{1}$ Este trabajo forma parte de la tesis doctoral "Representaciones Sociales de la maternidad temprana, en madres jóvenes", realizada en el Programa del Doctorado en Estudios Socioculturales de la Universidad Autónoma de Aguascalientes con financiamiento de CONACyT.

${ }^{2}$ Licenciada en Psicología (UAA), Maestra en Tecnología Educativa (ITESM) y Doctora en Estudios Socioculturales (UAA). Profesora del Doctorado en Ciencias de la Educación en Universidad Cuauhtémoc plantel Aguascalientes, México. Correo electrónico:

belgica.romero@edu.uaa.mx

(10) https://orcid.org/0000-0002-4029 7377

Para citar este artículo use: Romerode-Loera, B. (2018). Hallazgos y discursos en torno a la maternidad adolescente. Civilizar, 18(35). 163175. doi: 10.22518/usergioa/jour/ $\operatorname{ccsh} / 2018.2 / \mathrm{a} 12$
} 


\section{Introducción}

El objetivo del análisis presentado es el de una exploración del tema de la maternidad adolescente de tal manera que puedan ser visibles los debates en torno a este y con ello generar preguntas de investigación que sirvan a la formulación de proyectos que permitan robustecer el conocimiento de un tema social actual.

El proceso de revisión de esta investigación se realizó teniendo como criterio su indexación en las bases de datos EBSCO, SciELO, Redalyc, el índice de revistas mexicanas de investigación científica del CONACyT, así como en el motor de búsqueda Google Schoolar. Para la búsqueda se utilizaron las palabras "maternidad", "adolescencia" y "juventud" en sus diversas combinaciones en español e inglés, dando preferencia a los documentos publicados a partir del año 2000 considerándolos como actuales, concluyendo con aquellos publicados en el 2013, año en que inicia el proyecto de investigación del cual forma parte esta revisión. Una vez realizado el primer acercamiento se buscaron y analizaron otras publicaciones de manera focalizada a partir de su mención en las fuentes primarias, o bien aquellas que se acercaban más al contexto sobre el cual se desarrolló la posterior investigación, a saber, Aguascalientes, ciudad media del centro de México. En este último rubro ya no se consideró el criterio de indexación y se ubican los estudios de Román Pérez (2000), Stern (2003; 2007; 2012) y Núñez y Ayala (2012) como representativos de los estudios en México así como el ensayo de Padilla (2012) y el trabajo de Ortiz Lozano y Maza Díaz Cortés (2010) en Aguascalientes.

En resumen, los primeros criterios de búsqueda fueron revistas indexadas en las bases mencionadas y periodo de publicación 20002013. Posteriormente se realizó una segunda búsqueda seleccionando aquellos artículos de investigación que cumplieran alguno de los siguientes criterios: a) Investigaciones referidas en los artículos analizados en la primera búsqueda.

b) Investigaciones representativas de la maternidad adolescente en México hasta 2013.

c) Investigaciones representativas del contexto cercano en el que al momento de este trabajo, se pretendía comenzar una investigación, esto es la ciudad de Aguascalientes, ubicada en el centrooccidente de México. Sus características principales son que cuenta con una población mayoritariamente urbana, tradicionalista con fuerte connotación católica, presenta índices de calidad de vida por encima de la media mexicana y con una economía sustentada en la manufactura para grandes multinacionales, así como en las medianas y pequeñas empresas de servicios. Una característica destacable de este contexto es que a nivel de imagen social se refiere a sí misma como una ciudad de "madres adolescentes", a pesar de que hasta antes de 2013, la incidencia de nacimientos de madres menores de 20 años era menor al 20\% de total de nacimientos (INEGI, 2013) manteniéndose constante a lo largo de los años y no en aumento como se suele argumentar en los medios locales.

El análisis de las investigaciones encontradas se realizó a partir de los resultados más importantes reportados en cada estudio, para lo cual se organizaron en temas: salud, razones para ser madres, relación con educación; contexto y familia de origen, relación de pareja y crianza de los hijos, imagen de género, significación de la maternidad; políticas públicas; y representaciones sociales de la maternidad. Estos temas direccionaron los cuestionamientos de investigación ¿La maternidad en la adolescencia representa un riesgo para la salud del niño y/o la madre? ¿Cuáles son las razones de 
una joven para tener un bebé en la adolescencia? ¿La educación, contexto y la familia de origen de la joven pueden considerarse factores de predicción de una maternidad temprana? ¿Qué sabemos de la relación de pareja y crianza de los hijos de las adolescentes? ¿Cómo interviene la imagen de género en la maternidad temprana? ¿Cuál es el significado de la maternidad para las jóvenes? ¿Cómo son visibilizadas las madres adolescentes en las políticas públicas? ¿Cómo es representada socialmente la mujer y la maternidad? ¿Cómo se está investigando el tema?

Una segunda categorización de las investigaciones analizadas se da a partir de describir los cuerpos teóricos y diseños metodológicos desde donde se hace un acercamiento al fenómeno. Dado que no fue un criterio de selección el que estuviera de manifiesto la teoría que subyace a cada investigación, en algunos de los casos se hizo necesario inferirla a partir de los conceptos y descripciones hechas por los autores. La misma acción se siguió al analizar la metodología, en algunos casos descrita como diseño, como enfoque o simplemente en la mención de las herramientas empleadas para la recolección y análisis de la información.

\section{Resultados}

Los resultados se presentan describiendo los hallazgos de cada uno de los temas o categorías de análisis. Dado que es un análisis cualitativo el descrito, se privilegia la profundidad de la descripción, procurando poner en diálogo las investigaciones.

\section{Salud y maternidad temprana}

Un argumento que da cuenta de la representación de la maternidad de jóvenes utilizado para considerarlo como una problemática de salud pública, es el riesgo que corre la salud de la madre y el hijo. Este argumento es difundido principalmente en el sector salud a nivel local y nacional y podemos observar una muestra en los medios de comunicación, como en la decla- ración del titular de Programa de Planificación Familiar y Climaterio del Instituto de Servicios de Salud del Estado (ISEA), José Ríos Velasco al mencionar que:

[...] el embarazo en adolescentes conlleva riesgos, como desarrollar diabetes gestacional o enfermedad hipertensiva, que se convierte en preclamsia e incluso en eclampsia, así como ruptura de membranas que provocaría que el bebé nazca antes de que esté maduro, provocando muerte prenatal o perinatal, entre otros (Diario Rotativo, 4 Oct. de 2013).

Un ejemplo más de este argumento lo podemos observar en el informe sobre embarazo adolescente de la FNUAP (Loaiza y Liang, 2013) donde se establece como objetivos para la población joven el reducir la mortalidad infantil y mejorar la salud materna asociando dichos problemas a la maternidad temprana.

Algunas investigaciones realizadas desde el campo de la salud tienen por objetivo conocer las prácticas de los adolescentes en el uso de anticonceptivos, antes o después del parto. En su mayoría parten de la premisa de que el embarazo en esta etapa de la vida es consecuencia del desconocimiento o mal uso de métodos anticonceptivos. Al respecto existen resultados que muestran que las adolescentes de contextos urbanos refieren mayormente embarazos no deseados y a la vez más conocimiento sobre métodos anticonceptivos: pastillas, DIU y condón. Sin embargo, solo una tercera parte de las madres jóvenes utilizan algún método anticonceptivo después de 6 semanas del parto (Climent et al., 2000; Núñez-Urquiza, Hernández-Prado, García-Barrios, González y Walker, 2003), lo cual concuerda con la ambivalencia de las jóvenes en el uso de anticonceptivos encontrada en estudios de corte cualitativo (Bender, 2008; Wahn, Nissen y Ahlberg, 2005).

El conocimiento de los anticonceptivos pareciera no ser un problema, sin embargo, los datos de conocimiento no corresponden con los de uso adecuado, ya que la mayor dificultad 
es la puesta en práctica de esos conocimientos debido a la socialización de género que no les permite consultar acerca de ellos y tomar la iniciativa de su uso en la negociación con la pareja -concretamente respecto al preservativo o condón- (Climent, 2009). En este mismo tenor, el aborto aparece como una opción arriesgada e inaceptable.

Estas investigaciones suelen ver el embarazo en la adolescencia como un problema de salud pública, bajo supuestos de riesgos en la salud en la madre y el niño, aunque otras de las investigaciones muestran que los problemas a la salud no están relacionados con el embarazo sino con las condiciones de precariedad en que viven la mayor parte de las adolescentes embarazadas (Climent, 2003a; de la Cuesta, 2001; Stern, 2012). Incluso algunos riesgos de salud asociados al embarazo, parto y posparto, no son tales en las adolescentes, como el caso de que su densidad ósea es tres veces mayor al año de posparto que en las madres adultas (Sámano et al., 2011).

Entonces, aun cuando las razones para considerar la maternidad temprana como un problema de salud solamente son ciertas con respecto a las menores de 15 años, los problemas de salud asociados son transferidos a la representación social de la maternidad temprana a edades más avanzadas de la juventud, utilizando este argumento como uno de los principales en la promoción de acciones de planificación familiar y prevención de la maternidad adolescente, pero también contribuyen a su estigmatización como jóvenes irresponsables o descuidadas con la salud.

\section{Razones de las jóvenes para convertirse en madres}

En una investigación realizada en Suecia (Wahn et al., 2005), las jóvenes refieren como causas de su embarazo la falta de oportunidades, la ambivalencia en el uso de anticonceptivos y los patrones familiares de embarazos tempranos. Por su parte, en las investigaciones latinoamericanas (Climent, 2003a; 2009a; 2009b; Climent et al., 2000; de la Cuesta, 2001; Núñez-Urquiza et al., 2003; Pacheco-Sánchez et al., 2007; Román Pérez, 2000; Sámano et al., 2011; Stern, 2007; 2012) no se menciona la falta de oportunidades sino la falta de un proyecto de vida alterno, aunque al no existir oportunidades para las jóvenes no se plantean proyectos de vida realizables.

En todas las investigaciones que exploran los patrones familiares se destaca la presencia de un patrón familiar de embarazos tempranos, no como causa sino como factor relacionado. Por su parte, la mayor parte de las investigaciones realizadas desde la óptica social encuentran como razón principal de maternidad el deseo de serlo o como una consecuencia del "estar enamorada". Cabe destacar que las maternidades demasiado tempranas -menores de 15 años-, en mayor medida son resultado de actos de violencia sexual (Stern, 2012); por ello es importante diferenciar el embarazo y la maternidad que viven las mujeres menores de 15 años en un primer corte de edad. Entonces surge el cuestionamiento de ¿qué sucede con las jóvenes madres de 16 a 20 años de edad que desean la maternidad? Se habla de la prevención del embarazo no deseado o planeado como si fuesen sinónimos, pero ¿existe un deseo en estas jóvenes por convertirse en madres de manera temprana? ¿El deseo o aspiración a la maternidad es siempre consciente y racional?

\section{Educación, contexto y familia de las jóvenes}

La razón de conjuntar tres temáticas aparentemente diferentes en una misma categoría, obedece a la idea que manifiestan algunos académicos sociales sobre tratar de explicar la prevalencia del fenómeno en una historia familiar caracterizada por maternidades tempranas a manera de "estilo familiar" y en un contexto social desfavorecido y violento, mientras que se menciona como el efecto principal e inme- 
diato la desescolarización de las jóvenes. Es por ello que se decide explorar la forma en que las investigaciones analizadas abordan dichas temáticas.

A partir del estudio del embarazo adolescente en diferentes contextos, Stern (2007) encuentra que algunas conductas y subjetividades se presentan en mayor medida en un contexto social que en otro: En el contexto marginal, la relación entre estereotipos de género, las condiciones sociales (contexto) y aspiraciones de vida traen como consecuencias una iniciación sexual temprana, ausencia de protección, embarazos y nacimientos tempranos. En el sector popular se demora la maternidad más allá de los 18 años, los embarazos inesperados se dan dentro de noviazgos prolongados, donde el varón asume la paternidad. Por último, en el contexto clase media alta, la iniciación sexual es más temprana que las generaciones anteriores, los embarazos inesperados son ocultados o se recurre al aborto, privilegiando el proyecto de vida escolar.

Aun cuando se asocia la maternidad en la adolescencia a la pobreza, la escolaridad, la marginalidad y el desempleo, esta relación no es una causa directa sino un contexto para pautas de socialización que pueden tener efecto en el deseo o decisión de ser madre. Por ejemplo, en relación a la educación versus maternidad como proyecto de vida, en la familia se refuerzan los estereotipos de género que llevan a construir una autoestima baja en relación al estudio como proyecto de vida, mientras que también en el seno familiar se refuerzan idealizaciones sobre la maternidad (Climent, 2003a).

En relación con la familia de origen, una investigación realizada en Estados Unidos por Corona Garret y Tidwell (1999), mostró la diferencia entre madres adolescentes y adolescentes que no son madres, en donde las primeras presentan una relación conflictiva con sus madres. Así mismo, Román Pérez (2000) refiere que la dinámica familiar de muchas de las madres adolescentes está caracterizada por conflictos entre los padres y ellos. Climent (2009a) por su parte, analizó la forma en que las relaciones familiares, en especial los estilos parentales, la calidad de las relaciones familiares y la socialización de género promueven la formación de representaciones sociales acerca de los roles de género, la familia, la sexualidad y la maternidad, entre otros aspectos que van a estar presentes en los comportamientos reproductivos y en los proyectos de vida. Así, por ejemplo, en las familias con un estilo parental democrático, el embarazo deseado puede verse como proyecto de vida, encontrando con ello la identificación con modelos maternos, en el caso de los embarazos sorpresivos se deben a descuido en el uso de anticonceptivos. Ante un estilo parental permisivo, el embarazo es naturalizado, aceptado y hasta alentado, o bien se da por reglas laxas con respecto a la sexualidad. En las familias con un estilo autoritario-paternalista, en las cuales hay mayor número de conflictos entre los miembros de la familia,

el embarazo suele ser inesperado y conflictivo y puede leerse como un desafío a los límites rígidos y controles coercitivos, un modo de compensar carencias afectivas, una respuesta a información inadecuada en relación a la sexualidad y a dificultades de negociación con la pareja sobre tener o no relaciones sexuales e hijos (Climent, 2008).

En las familias negligentes-despóticas que suelen incluir la violencia familiar y muchos conflictos entre todos los miembros, los embarazos suelen verse como una respuesta a la incoherencia entre límites restrictivos y la falta de control, siendo interpretados como un castigo o llamado de atención a los padres.

Por último, con referencia al aspecto educativo, todas las investigaciones coinciden en que la deserción escolar de las adolescentes no necesariamente tuvo que ver con la maternidad, presentándose antes del embarazo en muchos de los casos. Este dato es confirmado con las estadísticas del contexto mexicano donde 
las madres tienen bajo nivel de escolaridad: el $64.9 \%$ de las mujeres que fueron madres en 2014 cuentan con escolaridad de secundaria o menor (INEGI, 2014). Así, aunque el embarazo no es causa de deserción escolar, la escuela parece sí ser un medio de protección para los jóvenes (Chaves, 2009), ya que existen asociaciones entre el nivel de escolaridad y el uso de anticonceptivos, postergación de la maternidad y espaciamiento entre los hijos (Chaves, 2009; Menkes y Suárez, 2003; Stern, 2007).

\section{Relación de pareja y crianza de los hijos}

La relación de pareja posterior al embarazo puede tener diferentes desenlaces. Entre las madres jóvenes marginadas es más frecuente que se embaracen siendo solteras, que las primeras uniones conyugales no sean planificadas y que tengan malas relaciones con la pareja. Hay mayor proporción de separaciones, casamientos sucesivos apresurados y violencia conyugal. Stern (2012), encontró que el desenlace de una relación posterior al embarazo adolescente está en relación al contexto en el cual está inmersa la pareja.

Llama la atención que la mayor parte de las investigaciones en torno al tema planteen reflexiones desde una perspectiva feminista, identificando el papel de los roles de género en diferentes contextos, pero casi ninguna aborda la relación de pareja, mucho menos el papel de la paternidad de los jóvenes: Román Pérez (2000), presenta a mayor profundidad la relación de noviazgo entre los adolescentes como preámbulo a la maternidad/paternidad, mientras que por su parte, Stern (2007) concluye sobre la diferencia de comportamiento. Pacheco-Sánchez et al. (2007), afirman que la socialización de género permite la diferenciación en significados respecto a la relación sexual entre jóvenes. Así para las jóvenes, la relación sexual se asocia a la reproducción y se vive en varios casos como algo negativo, mientras que para los jóvenes existe la posibilidad placentera de la relación sexual y es significada como forma de reafir- mar la masculinidad. La falta de investigaciones donde se incluye al joven puede deberse a que suele asociarse a la maternidad en menores de 20 años con la soltería cuando la mayoría se encuentra en pareja al momento del nacimiento del bebé y la mayoría con parejas de una edad similar a la suya, al menos en las de 16 a 20 años (Gogna, 2005).

Lo cierto es que, con las actividades de la crianza entre parejas adolescentes, los conflictos se agudizan debido a que tienen que adaptarse a nuevos roles de pareja y crianza que implican iniciar la adultez, lo cual no sucede de igual manera para la mujer que para el hombre. Para las mujeres ser madres las convierte en adultas puesto que la tradición les exige asumir el rol, mientras que muchos de los varones adolescentes son protegidos de asumir la responsabilidad de la paternidad por los padres, sobre todo en estratos sociales medios y altos (Maldonado y Micolta, 1999).

Con respecto a la crianza de los hijos, Maldonado y Micolta (1999) encuentran que entre las parejas adolescentes la crianza es asumida por la mujer y el varón realiza una función exigida de proveedor, sobre todo en los estratos sociales más pobres, mientras que en estratos sociales medios y altos la función del padre se excusa por ser adolescente. Ambos tienen la ilusión de proporcionar al hijo lo que no obtuvieron de sus propios padres. Las jóvenes ven la crianza de los hijos como un proceso doloroso y difícil y cuando las madres continúan estudiando o trabajan, se comparte la crianza con otros miembros de la familia, generalmente la madre o la abuela.

Podemos ver entonces, que los padres adolescentes han estado ausentes en la investigación de madres adolescentes, lo que nos lleva a preguntarnos si también estarán ausentes en la crianza de los hijos, y si es así ¿cómo vive la madre adolescente la presencia o ausencia de la pareja en relación con ella misma y con la crianza de los hijos? Así mismo, podemos ob- 
servar que existe poca investigación referente a la crianza de los hijos de madres adolescentes por lo cual nos quedan las preguntas ¿de dónde obtiene la información las jóvenes para la crianza de los hijos?, ¿cómo influye la información de la crianza de los hijos en la configuración de la representación social de la maternidad que construyen estas jóvenes?, ¿cómo influye el conocimiento sobre actividades de crianza en su sentido de autoeficacia con respecto a la maternidad?

\section{Imagen de género}

Con este concepto se hace referencia a la socialización de género manifestada en estereotipos para hombres y mujeres y la naturalización de la maternidad. Al respecto, Stern (2012) a partir del estudio de diferentes contextos mexicanos y cómo se presentan los estereotipos de género en cada uno de ellos, considera que la manera en que estos se presentan, lleva a los jóvenes a vivir de manera diferente la sexualidad y sus consecuencias, tales como el embarazo.

Aunque hay similitudes en los estereotipos de género y de cómo actúan en los tres sectores estudiados (marginal, popular y clase media alta) "los resultados de cuándo - a qué edad y en qué etapa de la vida-tienden a ocurrir los embarazos, su frecuencia relativa, si conducen a un matrimonio, a una unión, etc. son aparentemente muy diferentes en los tres sectores" (Stern 2007, p.126).

Otros autores como Pacheco Sánchez et al. (2007) y Worobiej (2008) encuentran también que la construcción cultural que se hace de la diferencia de género, marca los significados que se dan a la sexualidad, así las mujeres lo relacionan con el ejercicio reproductivo y los hombres con una forma de sustentar su masculinidad. El relacionar la imagen de género con la reproducción también está mediado por los proyectos de vida asociados a cada género en diferentes contextos, aunque en los diferentes contextos mexicanos, esta relación está presente como parte de la cultura.

\section{Significado de la maternidad en las jóvenes}

En esta categoría se ubican los resultados de estudios que indagan el significado o subjetividad que tiene la maternidad para las mismas madres jóvenes, al respecto en la investigación de Climent (2009a), sobre la representación de la maternidad adolescente, menciona que las adolescentes en su mayoría no lo ven como un problema sino que lo relativizan de acuerdo a cada caso; tal como ocurre en un investigación realizada en por Kirkman et al. (2001), donde las madres adolescentes entrevistadas en el contexto australiano, se identifican como buenas madres, que pueden mejorarse y con capacidad de afrontar las dificultades que se les presentan.

En las familias de madres adolescentes, donde existen varios casos, la maternidad adolescente es algo reconocido y estimado, incluso idealizado; para algunas madres, en contextos de marginación, la apariencia física del embarazo aumenta el estatus social. Por su parte, Llanes (2014), a través de la entrevista a madres adultas que en su momento fueron madres adolescentes, explora trayectorias de maternidad y su significación, encontrando que las estrategias de significación de la maternidad se yuxtaponen con la experiencia de la vida de pareja, y con el apoyo afectivo y económico de los padres.

Al describir estos resultados, la mayoría en contextos latinoamericanos, no se quiere señalar que la maternidad temprana se experimente siempre como placentera y deseada sino que la manera como las mujeres significan la maternidad, está influida por el contexto, la relación con sus padres, el proyecto de vida, las oportunidades y la concepción cultural (o representación social) de la maternidad. El interés se centra en cómo se realiza esta interiorización o subjetividad del contexto cultural (clase social, oportunidades, información) de las jóvenes con respecto a la maternidad. 


\section{Políticas públicas para madres adolescentes}

Dado que los argumentos para ver a la maternidad de las jóvenes como problema de salud pública vienen mayormente desde el sector salud, es ahí donde se han enfocado la mayor parte de las políticas públicas de prevención del fenómeno a través de los programas de salud reproductiva. Al respecto, Stern (2012) menciona que la mayoría de las necesidades de las madres jóvenes y jóvenes embarazadas, se encuentran fuera del sector educativo por lo que son necesarias políticas públicas integrales donde la educación informal, comunitaria y a través de los medios masivos de comunicación tomen un papel preponderante. También sugiere analizar la legislación respecto al aborto, la impunidad de los varones en la reproducción, el abuso sexual y la violencia de género, sobre los agentes de la salud y la planificación familiar.

Aun desde la salud hay varias tareas pendientes, como la promoción de técnicas anticonceptivas acordes a las características de la actividad sexual de los adolescentes (esporádica) y las socializaciones de género existentes en los diferentes contextos. Para ello es necesario reconocer y modificar la manera en que los agentes de la salud valoran la actividad sexual y reproductiva de los y las jóvenes. Adaszco (2005) por su parte, encuentra que los agentes de salud suelen tener representaciones del embarazo adolescente como un problema de salud pública, que lleva consigo riesgos para la salud del niño y de la madre, que impide a los jóvenes salir del "círculo de la pobreza"; pero las consecuencias del embarazo en las jóvenes son diferentes de acuerdo a los contextos, siendo en las clases bajas naturalizado y agravando las condiciones de vida precaria preexistentes, mientras que para las clases medias es más estigmatizado y la consecuencia principal es el abandono de un proyecto de vida escolar. Dicha representación creemos que parece ser reflejo de la representación social de la maternidad temprana que se tiene en América Latina.
En relación con lo anterior, Gogna (2005) concluye a partir de sus investigaciones sobre políticas púbicas dirigidas a prevenir la maternidad antes de los 20 años en Argentina, que algunas están permeadas por estereotipos de género y clase, y un sesgo adulto-céntrico, mientras que otras presentan mayor sensibilidad a la complejidad social detrás del embarazo a temprana edad. Rescatando la Idea de Furstenberg (2003), "la maternidad adolescente tiene que ver más con como nuestra cultura política ha respondido a los problemas asociados con la pobreza, las relaciones de género y cosas parecidas" (Citado por Adaszco, 2005 p.36). Creemos que dentro de "esas cosas parecidas" se puede encontrar la significación o representación social de la maternidad en las jóvenes.

\section{Representaciones sociales de la maternidad}

Los estudios en representaciones sociales de la mujer revelan la centralidad de la maternidad dentro de dichas representaciones, ya sea que estas sean analizadas y recogidas en los instrumentos sociales tales como el arte (Álvaro Estramiana y Fernández Ruiz, 2006), o en los discursos de las mismas mujeres (G. Climent, 2009a; Política y Canavate, 1999; Schwarz, 2005). Para Álvaro Estramiana y Fernández Ruiz (2006) las representaciones pictóricas de la mujer a través de la imagen del cuerpo femenino ha naturalizado el orden social naturalizando valores y virtudes morales en el cuerpo de la mujer y con ello buscando un control del cuerpo social. Estos investigadores mencionan que la mujer ha sido representada pictóricamente como símbolo de la reproducción y fecundidad, como marca de vicios y efectos y como desviación y marginalidad. Como símbolo de fecundidad, la mujer es representada eliminando su sensualidad y mostrando sus órganos diseccionados, o bien dentro del rol de la crianza y cuidado de los hijos; estas representaciones muestran la centralidad de la representación del cuerpo de la mujer fecundo o en rol de madre dentro de los valores de la sociedad. 
En general, los autores resaltan las representaciones pictóricas del cuerpo femenino como la forma de objetivación del orden social, teniendo así que todos los valores sociales y sus transgresiones son representados en un cuerpo de mujer o en sus partes diseccionadas, lo que permite a las sociedades naturalizar el orden social al darle una imagen física.

Por su parte Schwarz (2005), señala que las decisiones en torno a la maternidad afectan a la sociedad en su conjunto y en su desarrollo ya sea solamente en demografía como en las pautas de socialización de las nuevas generaciones. Aunque recoge información de mujeres que no son madres y por ende no tienen vivencia de ello, identifica que el rol de madre en el discurso de las mujeres, aparece como parte central del núcleo de la representación social de la mujer, presentándose como exigencia para la mujer, pero aludiendo a ejemplos cercanos para mostrar que llegado el momento, la mujer podría con todas estas exigencias o roles, mientras que el trabajo aparece como conexión con la maternidad al afirmar que una mujer trabajadora trae beneficios para el hijo.

Concretamente, en las representaciones sociales que tienen las madres jóvenes y que han sido analizadas recientemente en dos investigaciones paradigmáticas: la de Política y Canavate (1999) en Colombia y la de Climent (2009a) en Argentina, aparecen el nuevo status social, el miedo al rechazo familiar, las decisiones trascendentales, la postergación de estudios, el padre del bebé como proveedor y la dependencia familiar y económica, como contenidos de la representación social de la maternidad en las jóvenes. En el caso de las madres multigestantes, aparecen también el padre del hijo como contenedor emocional y el bebé como fuente de amor.

En la investigación de Climent (2009a) la polaridad de los elementos determina si en las representaciones sociales de las jóvenes y en las hegemónicas (sociedad) el embarazo y maternidad adolescente es visto como problema o no. En muchos de los caso la maternidad "es vista como una búsqueda de afirmación social $\mathrm{y}$ afecto, una forma de realización personal que incluso eleva la autoestima, presentando así una connotación positiva del embarazo" (Política y Canavate, 1999, p. 196); lo que convierte a la maternidad de éstas jóvenes en parte central de su proyecto de vida y en algunas ocasiones en la única función de la mujer.

\section{Estrategias metodológicas y cuerpos teóricos de investigaciones en maternidad temprana}

Con excepción de las investigaciones completamente desde un enfoque de salud pública y con metodologías cuantitativas (Sámano et al., 2011; Alonso et al., 2005; Núñez-Urquiza 2003; Quezada-Salazar, 2008), el resto de las investigaciones se llevan a cabo desde un paradigma cualitativo bajo los enfoques etnográfico (Stern, 2003), interpretativo (Pacheco et al., 2003; Miño-Worobiej, 2008; Smith Battle, 2000) y teoría fundamentada (De la Cuesta, 2001) decantándose en la mayor parte de los estudios por la técnicas de entrevista a profundidad (Stern, 2003; Climent, Arias y Spuro, 2000; Climent, 2003, 2009, Miño-Worobiej, 2008; Pavan, 2001; Bender, 2008; Yardley, 2008; Molborn y Jacobs, 2011; Corona Garret y Tidwell, 1999; Núnez et al., 2012; Padi1la, 2012; Maldonado y Micolta, 1999), observación (Stern, 2003), relatos biográficos (Stern, 2003; Kirkman, 2001) y grupos focales (Pacheco et al., 2003, Gogna, 2005), utilizando el análisis de contenido como técnica de análisis de la información. Los estudios que atienden a mayores elementos del fenómeno como contextuales, significados, estilos parentales, relaciones de pareja, son aquellos que utilizan diversas técnicas de recolección de datos y utilizan una metodología interpretativa.

Dejando de lado los estudios de salud reproductiva en los cuales el enfoque es el embarazo adolescente como un riesgo de 
salud pública, los estudios sociales están mayormente realizados desde un enfoque de significaciones desde los sujetos, algunos bajo la teoría de las representaciones sociales (G. Climent, 2009b; Gómez-Sotelo et al., 2012), o el interaccionismo simbólico (de la Cuesta, 2001) y la mayoría desde un enfoque de género (Pacheco et al,. 2007; Worobiej, 2008; de la Cuesta, 2001, Climent, 2003,2009, Núñez et al., 2012; Padilla, 2012; Román Pérez, 2000); aun cuando varios no lo hagan explícito sí se observa esta perspectiva en sus conclusiones. La perspectiva de género parece una recurrencia natural debido a que se parte de la maternidad y la juventud o adolescencia como categorías socialmente construidas y deconstruidas desde una filosofía feminista. Con respecto a los estudios de género, Saletti (2008) identifica dos tipos de posturas feministas en relación a la maternidad; aquellas cuyo objetivo es el de desarticular el modelo de la buena madre, deconstruyendo el instinto maternal o la maternidad como identidad femenina y las que buscan construir la maternidad como fuente de placer, conocimiento y poder específicamente femeninos.

Parece ser que el enfoque de género adoptado en la mayoría de las investigaciones analizadas parten del supuesto feminista de cuestionar la maternidad como identidad femenina y analizar la mayor parte de constructos contextuales en la significación y vivencia de la maternidad en la adolescencia desde una perspectiva de género que analiza las formas en que el poder masculino ha influido en la vida de las mujeres. Puede considerarse que algunos de los principios de la perspectiva de género son indispensables en cualquier investigación que toque la realidad de las jóvenes, y más cuando el centro está en las mujeres como madres en un contexto socio-histórico androcéntrico. Por lo tanto, es deseable que la investigación sobre madres jóvenes debería incluir el análisis crítico de los procesos sociales de normalización contextuales y las agencias que hacen las jóvenes sobre ellas, y que moldean y transforman las experiencias de la maternidad.

\section{Conclusiones}

De las investigaciones analizadas en esta revisión, podemos retomar como antecedentes para posteriores investigaciones, que el embarazo en la adolescencia suele verse como problema social debido, según Román Pérez (2000), ya que rompe con una expectativa que los adultos, principalmente los padres, tienen sobre la juventud como una etapa de preparación para la vida adulta. En este sentido, Climent (2003) considera que al plantearse la maternidad adolescente como un problema social es necesario preguntarse, si es una pauta cultural que debe respetarse o si es un evento de consecuencias indeseables para la vida de las mujeres y sus familias que debe prevenirse, razón por lo cual es necesario ahondar en las funciones o carencias que suple la maternidad en estas jóvenes. Desde una perspectiva de género, hace falta ahondar sobre la relación potencial de las mujeres con el poder de la reproducción y de los hijos desde una visión que rescate la experiencia de la maternidad como fuente de placer y amor.

Resulta también fundamental profundizar en el estudio del noviazgo entre los adolescentes, ya que es la situación en la cual las adolescentes "llegaron a una relación coital que adquirió carta de naturalización en aras del amor, de promesas de matrimonio y/o de deseos de maternidad/paternidad" (Román Pérez, 2000 p. 193). En sí la profundidad carente es en la relación de pareja y el papel del padre adolescente en la experiencia de la maternidad en las jóvenes.

Otro punto poco explorado es el de la vivencia del embarazo, desde el coito, su sospecha, el reclamo, el perdón y el apoyo casi siempre por parte de la familia y la continuación o no de la relación con la pareja. En las investigaciones revisadas solamente tocan este tema Román Pérez (2000), Gómez-Sotelo et al. (2012) y Padilla (2012). Hace falta ahondar en la práctica de las relaciones sexuales antes del embarazo con un objetivo comprensivo hacia la 
relación que establecen las adolescentes entre sexualidad y reproducción surgido de la forma en que la cultura toma el impulso sexual y lo transforma en lo maternal, dándole un fin determinado y único (Saletti Cuesta, 2008).

Por último, es necesario profundizar a través de investigaciones que aborden a la maternidad en la vida cotidiana, desde las actividades que implican la crianza de los hijos, las aspiraciones que tienen las jóvenes con respecto a sus vidas y las de sus hijos. Es en este último debate donde se inserta nuestra investigación: la maternidad en la juventud desde la conformación del sentido común (representaciones sociales) y sus implicaciones en la vida cotidiana de la crianza de los hijos.

A partir de la información analizada en torno al embarazo y maternidad en la juventud, surgen preguntas como ¿cuál es la representación social que tienen las jóvenes madres sobre la maternidad? ¿Quiénes influyen en la información sobre maternidad en la vida de las jóvenes? ¿A qué problemáticas se enfrentan? ¿Continúan en las relaciones de pareja? ¿Cuál es el papel de la pareja? ¿Cómo es la vida cotidiana de la crianza de los hijos y sus jóvenes madres? ¿Cómo se vive o no la anticoncepción posparto? ¿Cuáles son las aspiraciones de vida de una madre menor de 20 años? ¿La maternidad para ellas trae beneficios? ¿Es un problema para ellas la maternidad en la juventud?

Parafraseando a Climent (2003), el que una mujer no estudie y más si ésta es una mujer embarazada, no parece nada raro y parece importarle poco al Estado y a la sociedad debido a que culturalmente se entiende el rol de la madre, independientemente de la edad, como una forma de autorrealización, y aunque ciertamente puede serlo para muchas mujeres, también puede no serlo para aquellas madres que no tienen la oportunidad de mejorar sus condiciones de vida precisamente por falta de oportunidades que les permitan construir proyectos de vida realizables. No solamente la educación, sino cualquier forma de participación social de la que las madres jóvenes y sus hijos queden excluidos, representan un foco de marginación en sus comunidades. Estudiar el fenómeno de la maternidad a edades tempranas desde las representaciones sociales de las mujeres que viven esta situación, facilita la comprensión de la manera en que las jóvenes subjetivizan la información referente a la crianza de los hijos y la manifiestan en pensamientos, opiniones, actitudes y conductas conformando una representación social de la maternidad o sentido común que guía su acciones en la vida cotidiana. Creemos que el acercamiento a esta comprensión permite determinar si la maternidad en la juventud debe considerarse como un problema y en cuyo caso, ¿para quién es un problema y por qué?.

En lo teórico, el hacer una investigación de la maternidad temprana desde algunos principios del Interaccionismo Simbólico y haciendo uso del concepto de Representaciones Sociales, permite aportar elementos para responder a las grandes preguntas sociales de ¿cómo interviene lo social en la elaboración psicológica, en este caso de la maternidad? y ¿cómo interviene esta elaboración psicológica (representación social de la maternidad temprana) en lo social?.

\section{Referencias}

Adaszco, A. (2005). El embarazo durante la adolescencia en las miradas de los funcionarios públicos. En M. Gogna (Ed.), Embarazo y maternidad en la adolescencia. Estereotipos, evidencias y propuestas para políticas públicas (pp. 112-161). Buenos Aires: CEDES.

Álvaro Estramiana, J. L., y Fernández Ruiz, B. (2006). Representaciones sociales de la mujer. Athenea Digital-Revista de pensamiento e investigación social, 1(9), 65-77.

Bender, S. (2008). Three cases of adolescent childbearing decision-making: the importance of ambivalence. Adolescence, 43. 
Chaves, M. (Ed.). (2009). Estado del arte. Investigación sobre sexualidad y derechos en la Argentina (1990-2002). Papeles de Trabajo, 2(5). Recuperado de: http://www.bioeticas.org/IMG/pdf/ gogna02.pdf

Climent, G. (2003). La maternidad adolescente, una expresión de la cuestión social. El interjuego entre la exclusión social, la construcción de la subjetividad y las políticas públicas. Revista Argentina de Sociología, 1(1), 77-93.

Climent, G. (2009a). Representaciones sociales sobre el embarazo y el aborto en la adolescencia: perspectivas de las adolescentes embarazadas. Cuadernos de la Facultad de Humanidades y Ciencias Sociales- Universidad Nacional de Jujuy, (37), 221-242.

Climent, G. (2009b). Voces, silencios y gritos:los significados el embarazo en la adolescencia y los estilos parentales educativos. Revista Argentina de Sociología, 7(12/13), 186-213.

Climent, G. I., Arias, D., y Spurio, C. (2000). Maternidad adolescente: un camino hacia la marginación. Cuadernos Médicos Sociales, 77(2), 81-97.

De la Cuesta, C. (2001). Contexto del embarazo en la adolescencia. Nos hicimos novios y ahí empezó todo. Revista ROL de Enfermería, 24(9), 584-590.

Diario Rotativo (4 Oct. de 2013). De adolescentes, uno de cada cuatro embarazos en Aguascalientes. Recuperado de: http://www.rotativo.com.mx/vida/ salud/162599-de-adolescentesuno-de-cada-cuatro-embarazos-enaguascalientes/

Gómez-Sotelo, Á., Gutiérrez-Malaver, M. E., Izzedin-Bouquet, R., Sánchez-Martínez, L. M., Herrera-Medina, N. E., y Ballesteros-Cabrera, M. (2012). Representacio- nes sociales del embarazo y la maternidad en adolescentes primigestantes y multigestantes en Bogotá. Revista de Salud Pública, 14(2), 189-199.

INEGI (2013). Encuesta de población, hogares y vivienda. Instituto Nacional de Estadistica Geografía e Informática: México.

INEGI (2014). Distribución porcentual de los nacimientos registrados según escolaridad de la madre, 1990 a 2014. Instituto Nacional de Estadística Geografía e Informática: México.

Kirkman, M., Harrison, L., Hillier, L., y Pyett, P. (2001). «I know I'm doing a good job»: canonical and autobiographical narratives of teenagers mothers. Culture, Health \& Sexuality, 3(3), 279-294. doi: 10.1080/13691050010026097

Loaiza, E., y Liang, M. (2013). ADOLESCENT PREGNANCY: A Review of the evidence. New York: UNFPA. Recuperado de: http://www.unfpa.org/webdav/ site/global/shared/swp2013/ ADOLESCENT\%20PREGNANCY UNFPA.pdf

Maldonado, M. C. M., y Micolta, C. (1999). Los procesos vitales cruzados en padres y madres adolescentes. Investigación \& Desarrollo, (9) 47-60. Recuperado de: https://search.proquest.com/openview/bd 92b3c57690609d425f3609f71b1c99/1?c bl $=2027442 \&$ pq-origsite $=$ gscholar

Menkes, C., y Suárez, L. (2003). Sexualidad y embarazo adolescente en México. Papeles de población, 35, 233-262. Recuperado de: http://www.scielo.org.mx/scielo. php?script $=$ sci arttext\&pid $=$ S140574252003000100011\&lng=es\&tlng=es

Núñez Noriega, G., y Ayala Valenzuela, D. J. (2012). Embarazo adolescente en el noroeste de México: Entre la tradición y 
la modernidad. Culturales, 8(15), 7-46. Recuperado de:

http://culturales.uabc.mx/index.php/

Culturales/article/view/128

Núñez-Urquiza, R. M., Hernández-Prado, B., García-Barrios, C., González, D., y Walker, D. (2003). Embarazo no deseado en adolescentes y utilización de métodos anticonceptivos posparto. Salud Pública de México, 45(Suplemento 1), 92-102.

Ortiz Lazcano, D., y Maza Díaz Cortés, O. (2010). Madres solas y adolescentes en Aguascalientes. Aportaciones desde la perspectiva de género para el diseño de políticas públicas. Aguascalientes, México: Instituto Aguascalentense de las Mujeres. Recuperado de: http://cedoc.inmujeres.gob.mx/ftpg/ Aguascalientes/agsmeta8.pdf

Pacheco-Sánchez, C. I., Rincón-Suárez, L. J., Guevara, E. E., Latorre-Santos, C., Enriquez-Guerrero, C., y Nieto-Olivar, J. M. (2007). Significaciones de la sexualidad y salud reproductiva en adolescentes de Bogotá. Salud Pública de México, 49(1), 45-51.

Padilla, Y. (2012). Ensayo sobre maternidad temprana en Aguascalientes en el 2012. Universidad Autónoma de Aguascalientes.

Política, R., y Canavate, D. L. (1999). Representaciones sociales de maternidad y paternidad en cinco ciudades colombianas. Recuperado de: http://www.primerainfancialac. org/documentos/all/documentos/ pdf/3LAMUS-representacionesmaternidad-paternidad.pdf

Román Pérez, R. (2000). Del primer vals al primer bebé. Vivencias del embarazo en las jóvenes. México: CIEUV-IMJ.

Saletti Cuesta, L. (2008). Propuestas Teóricas Feministas en relación al Concepto de Materidad. CLEPSYDRA, 7, 169-183.
Sámano, R., Morales, R. M., Flores-García, A., Lira, J., Isoard, F., de Santiago, S., y Casanueva, E. (2011). Las adolescentes no pierden densidad mineral ósea en el posparto: estudio comparativo con adultas. Salud Pública de México, 53(1), 1-10.

Schwarz, P. (2005). Influencia de las representaciones sociales de la maternidad en la construcción de identidad femenina en mujeres jóvenes de clase media urbana. Recuperado de: http://webiigg.sociales.uba.ar/iigg/jovenes investigadores/3JornadasJovenes/ Templates/Eje\%20identidad-alteridad/ Schwarz-identidad.pdf

Stern, C. (2003). Significado e implicaciones del embarazo adolescente en distintos contextos socioculturales de México: reseña de un proyecto en proceso. Estudios Sociológicos XXI, 63, 725-745.

Stern, C. (2007). Estereotipos de género, relaciones sexuales y embarazo adolescente en la vida de jóvenes de diferentes contextos socioculturales de México. Estudios Sociológicos XXV, 73, 105-129.

Stern, C. (2012). El «problema» del embarazo adolescente: contribuciones a un debate. El Colegio de México AC.

Wahn, E., Nissen, E., y Ahlberg, B. (2005). Becoming and Being a Teenage Mother: How Teenage Girls in South Western Sweden View Their Situation. Health Care for Women International, 26, 591603. doi: 10.1080/07399330591004917

Worobiej, M. (2008). Imágenes de género y conducta sexual y reproductiva. Salud Pública de México, 50, 17-31.

Yardley, E. (2008). Teenage mothers' experiences of stigma. Journal of Youth Studies, 11(6), 671-684. doi: $10.1080 / 13676260802392940$ 
